\title{
Design And Optimization of Integrated Mach Zehnder Modulators In X-Cut Lithium Niobate Thin Film
}

\section{Luma Z. Mohammed}

UOT: University of Technology

Makram A Fakhri ( $\square$ mokaram_76@yahoo.com )

University of Technology.Iraq https://orcid.org/0000-0001-9010-6121

\section{A. K. Abass}

UOT: University of Technology

\section{Evan T. Salim}

UOT: University of Technology

U. Hashim

UniMAP: Universiti Malaysia Perlis

\section{Research Article}

Keywords: integrated electro-optic modulator, lithium niobate films, effective mode area, Mach-Zehnder

Posted Date: August 11th, 2021

DOI: https://doi.org/10.21203/rs.3.rs-727479/v1

License: (9) This work is licensed under a Creative Commons Attribution 4.0 International License. Read Full License 


\section{Abstract}

The Mach-Zehnder electro-optic modulator (MZM) was designed and fabricated using the AFM COMSOL program and the pulse laser deposition (PLD) technique. The PLD technique was used to deposit silicon, silicon oxide and LN thin film. Previously estimated optical properties of the prepared films were employed in the theoretical design of the modulator. Optimum device parameters were obtained, which delivered a considerable figure of merit ( $(\mathrm{V}$.L) values within the range of 2.12 to 2.25 at a device length of $2 \mathrm{~cm}$.

\section{Introduction}

Throughout the past three decades, as the demand for telecommunication facilities increased and the subsequent need for large bandwidths grew accordingly, the need to compensate external modulation in the transmission of fiber-optic arrangements has intensified [1-3]. Within optical transmission systems, an optical modulator is a key component and building block that serves as an information encoding device starting in the electrical to optical domains. Hence, a relatively high-speed optical modulator is considered one of the key features of a successful optical transmission system [3]. Optical modulation depends primarily on free carrier dispersion effects. However, free carrier dispersions in the silicon optical modulation are fundamentally absorptive and nonlinear, thereby resulting in the degradation of the amplitude of optical modulation that might lead to signal distortion. In this work, a number of attempts are proposed to realise a high-performance optical modulator by using a variety of material/nanomaterial platforms to address any issues [4-9].

Among the utilised materials, lithium niobate $\left(\mathrm{LiNbO}_{3}\right)$ is the most preferred material because of its outstanding electro-optic modulation (EOM) features. A lithium niobate (LN) modulator obtains exceptional results for high-data-rate multilevel signal generation. A conventional LN modulator is typically designed using a low-indexed-contrast waveguide (WG) alongside weak optical confinement, whereby the utilised electrodes should be positioned at a relatively far distance from the optical WG. This approach is mainly employed to minimise the absorption loss and thereby results in an augmented drive voltage. Thus, the conventional LN modulator performs low productivity modulation and has a bulky size $[10,11]$.

Silicon photonics are emerging as a competitive platform on silicon-on-insulator (SOI) technology, showing great promise for next-generation photonic integrated circuits that has the advantages of optical interconnects [12]. Silicon photonics are becoming a valuable approach for future optical interconnection development mainly due to its high bandwidth, high-density I/O capability, feasibility of dense integration and correlated compatibility with the processing of complementary metal-oxide-semiconductor CMOS. Its high bandwidth is mainly attributed to compacted WG dimensions. Si integrated photonics deliver large bandwidth and subsequently low power within intra-chip and on-chip optical interconnections [13]. For nearly a decade, LN has been the most preferred material for optical modulator design because of its

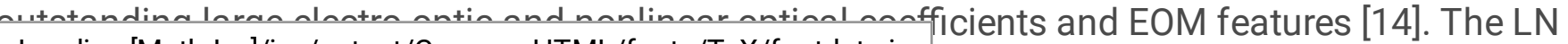
Loading [MathJax]/jax/output/CommonHTML/fonts/TeX/fontdata.js 
modulator shows good performance in high-bandwidth multilevel signal generation, low loss and low drive voltage [15]. Lately, the use of LN membrane on insulators has shown a potential pathway to produce a WG device that possesses good confinements. Furthermore, the hybrid design of silicon/LN (Si/LN) materials combines Si scalability alongside outstanding LN modulation efficiency. The proposed system relies on a super-mode WG structure that consists of $\mathrm{Si}$ and non-patterned LN membrane. This configuration helps optical mode distribution. However, it also decreases the efficiency of the modulation, which results in low electro-optic bandwidth and/or relatively high operating voltage $[16,17]$. Furthermore, the EOM in this geometry is considered a phase modulator. However, the stated phase modulator is known to be a component in an interferometric geometry of the so-called Mach-Zehnder modulator (MZM), in which the phase modulation in every single arm of the geometry is utilised to provide the required interference, thereby efficiently modulating the optical intensity $[18,19]$.

The main design concept is the utilisation of relatively high-index Si (WG), wavelength $(\lambda)$ of 1550 nmand refractive index at around 3.48. This condition can deliver an optical mode alongside close horizontal confinement, which results in a constant guided mode during the actual electro-optic modulation performance through the $L N$ thin film, thereby possibly providing the Si with a confinement modal. In contrast to the effect of carrier dispersion in the Si, the Pockels effect hypothetically tolerates an extreme broadband device alongside a maintained low optical propagation loss [20]. The voltage-length product $(V \pi . L)$ is a well-known figure of merit that can be employed to elaborate the inverse correlation between the voltage needed to modulate the optical intensity as an output of MZM from its maximum to minimum values through the application of $\pi$ phase shift (defined as $V \pi$ ) and the modulation region physical length L) [21]. Another important figure of merit is 3-dBe, which was also illustrated. The simulated MZM was introduced firstly to validate its higher performance and to investigate new possibilities of the fabricated MZM device. The dimensions that were considered firstly in the design are within the millimetre scale mainly because of the wire-cut dimensions used in the experimental work [22].

This work presents a geometrical simulation of a hybrid Si/LN MZM on the basis of the experimental results. The performance/efficiency, optimum dimension, confinement factor and the effective mode area to satisfy the single mode conditions are demonstrated.

\section{Design And Fabrication}

\subsection{Optical constant estimation}

The optical properties of the deposited films are investigated in the ultraviolet to near-IR wavelength to find the optical reflection of the deposited films by using (double-beam UV-vis) spectrophotometer of (Shimadzu UV-vis 1800, Japan) type. The refractive index (n) of the deposited materials was calculated using a developed equation $[23,24]$.

$\mathrm{n}=\mathrm{n}_{\mathrm{s}}\left(\frac{1+\sqrt{\mathrm{R}}}{1-\sqrt{\mathrm{R}}}\right)^{1 / 2}$. 


\section{2 theoretical wave guide design}

The MZM modulator design was obtained by employing the estimated optical properties from the experimental part. AFM COMSOL Multiphasic was used to simulate the optical mode and mesh the geometry of the WG. This algorithm can adapt to the arbitrary WG structure, obtain the effective mode indices and the effective mode area distribution in the WG, solve the bending loss and the mode distribution, and enable low V $\pi$.L and low metal absorption loss [15, 25]. Figure 1 shows a schematic of the investigated device.

\section{Results And Discussion}

The UV-Vis analysis of the deposited films on the LN-substrate is presented in Fig. 2(a) within the range of $200 \mathrm{~nm}$ to $800 \mathrm{~nm}$; this analysis was accomplished in accordance with the optical reflectance results. The reflectance spectrum of the deposited film tends to appear at around $320 \mathrm{~nm}$. A pronounced increment in the reflectance spectrum was observed with the wavelength increment up to $400 \mathrm{~nm}$. The reflectance spectrum of the deposited film was observed to be steady alongside the wavelength increment to $800 \mathrm{~nm}$ (within the visible region). A similar behaviour was previously reported in the literature $[26,27]$.

Concurrently, the reflectance spectrum of the deposited film on the Si-substrate with a wavelength range from $200 \mathrm{~nm}$ to $800 \mathrm{~nm}$ is outlined in Fig. 2(b). The optical reflectance was noted at around $270 \mathrm{~nm}$ in the ultraviolet region, after which a strong increment in the corresponding spectra was perceived after the stated wavelength up to $400 \mathrm{~nm}$. Moreover, within the visible region $(400 \mathrm{~nm}-800 \mathrm{~nm})$, the optical reflectance exhibited a steady behaviour. The results are in agreement with previously reported findings [27-30]. However, Fig. 2(c) illustrates a comparison between the Si substrate and LN substrate, showing that the deposited film on the LN substrate exhibited a higher reflectance tendency than that deposited on Si substrate (blue circled region) with respect to the wavelength increment. This finding clearly indicates the occurrence of a bathochromic shift in the reflectance spectrum of the deposited film on the LN substrate. This particular observation was noticed within the ultraviolet region. In the visible light region, the deposited film on the LN substrate also exhibited higher reflectance intensity than the one deposited on the Si substrate; this condition is referred to as a hyperchromic shift. An almost similar behaviour was observed in the mentioned wavelength region (red circled region).

The obtained experimental results were employed in the theoretical simulation were optimum modulator dimensions were decided. Figure $3(a$ and $b$ ), represents the effective index obtained at SiO2 thickness of $0.5 \mathrm{~mm}$. The increment started to appear at WG thickness of 0.041 and $0.039(\mathrm{~mm})$ for "without and with LN layer addition", respectively. Constantly, the effective mode area showed an increment profile with the increment of WG thickness. The confinements of the simulated MZM and MZM-LN are elaborated in the inset of Fig. 3 ( $a$ and $b)$. 
From the experimental results used in the theoretical simulation, the optimum modulator dimensions were determined. Figures $3(\mathrm{a}$ and $\mathrm{b})$ present the effective index obtained at $\mathrm{SiO}_{2}$ thickness of $0.5 \mathrm{~mm}$. The increment started to appear at WG thickness of 0.041 and $0.039(\mathrm{~mm})$ without and with LN layer addition, respectively. The effective mode area showed an increment profile with the increment of WG thickness. The confinements of the simulated MZM and MZM-LN are elaborated in the inset of Figs. 3(a and $b$ ).

With the limitation of this study concerning the available laboratory equipment, the thickness of the WG was high and therefore cannot be applied in the experiment because of the required high number of laser pulses (10,000 pulses and above), possible crack occurrence and mismatching.

The second design was proposed considering micrometre dimensions and was accomplished by multiplying by a factor of $10^{4}$. The micrometre MZM based on Si WG design with a variety of thicknesses and widths is demonstrated. The cross section of the $\mathrm{SiO}_{2}$ layer measured $100 \mu \mathrm{m}$, with thicknesses of $0.4,0.45$ and $0.5 \mu \mathrm{m}$. The $L N$ thin film was also added with a thickness of $100 \mathrm{~nm}$. The size of the utilised Si WG was in the range of $0.7 \mu \mathrm{m}$ to $3 \mu \mathrm{m}$, as shown in Figs. $4(\mathrm{a}, \mathrm{b})$. This figure effectively demonstrates the index and effective mode area alongside the increment in the Si WG width and thickness at $0.5 \mu \mathrm{m}$ $\mathrm{SiO}_{2}$ layer thickness for the simulated MZM and MZM-LN, respectively. The effective index tended to increase as the thickness and width of the Si WG increased, as observed in both simulated MZM and MZM-LN. However, upon the addition of the LN layer, the effective index values decreased due to the high refractive index of the deposited $L N$ nanostructure, thereby negatively affecting the effective index values, along with the very small particle size of the LND nanofilm [31]. Furthermore, the effective mode area increased in both MZM and MZM-LN devices alongside the Si WG width increment. In contrast, the effective mode area exhibited a reduction in the acquired values as the Si WG thickness increased. In the confinement variation, as shown in the inset of Figs. 4(c and d), high confinement was noticed at a low Si WG width, while a high-order mode and low confinement were observed at a larger width.

Finally, after the MZM fabrication, the refractive indexes of the Si and LN substrates as obtained from the optical analysis are utilised in the MZM simulation again to determine the effective index and effective mode area of the fabricated devices. The figure of merit $(V \pi$. L) as a function of the Si WG width and effective index is also demonstrated. Another important figure of merit, namely, 3-dBe, was also illustrated. For comparison purposes, the effective index and effective mode area of the simulated MZM$\mathrm{LN}$ device (SiO2 thickness $=0.5 \mu \mathrm{m}$ ) are replotted alongside the effective index and effective mode area of the fabricated MZM-LN device. Figures $5(a, b, c, d)$ presents the effective index and the effective mode area of the fabricated and simulated MZM-LN device using $\mathrm{LN}$ substrate at $\mathrm{SiO}_{2}(\mathrm{n}=1.44)$ and $\mathrm{LN}(\mathrm{n}=$ 2.346) at a thickness of $0.5 \mu \mathrm{m}$ and $100 \mathrm{~nm}$, respectively. The Si WG width and thickness varied in this investigation, and the refractive indices were 3.48 and 3.6111 for the simulated and fabricated MZM-LN, respectively. As demonstrated in Fig. 5(a), the effective index demonstrated higher values than those obtained using the bulk refractive index (Fig. 5[b]) possibly because the refractive index difference $(\Delta \mathrm{n})$ between the core and clad is higher in the fabricated MZM-LN than in the simulated MZM-LN. 
Furthermore, a high-order mode was obtained in the effective index of the fabricated MZM-LN. The effective mode areas of both simulated and fabricated devices are elaborated in Figs. 5(a) and 5(b), respectively. At a low Si WG thickness $(0.43 \mu \mathrm{m})$ of the fabricated MZM-LN, the TE mode is outside the WG geometry due to a high attenuation coefficient [24]. The high-order mode attained in the fabricated MZM-LN could be due to the newly applied refractive index (3.6111), which is higher than that applied in the simulated MZM-LN (3.48). This finding means that the fabricated MZM-LN has a higher effective mode area and in turn lower confinement as compared with that obtained in the simulated MZM-LN (inset of Fig. 5).

Figure 6 illustrates the fabricated and simulated MZM-LN device in terms of effective index ( $a$ and $b$ ) and effective mode area ( $c$ and d), respectively, using the Si substrate. The same parameters applied in Fig. 4 are applied in Fig. 6. However, the refractive index introduced to evaluate both the effective index and effective mode area is $n=6.101$. Figures $6(a$ and $b)$ show a clear increment in the effective index for the fabricated MZM-LN as compared with that obtained in the simulated MZM-LN $(n=3.48)$. In addition, the high-order mode obtained in the fabricated MZM-LN can be observed at width and thickness as low as 1.5 and $0.43 \mu \mathrm{m}$, respectively. However, a single mode was found at Si WG thickness of $0.44 \mu \mathrm{m}$, while a single mode was found at Si WG thickness from $0.45 \mu \mathrm{m}$ up to a WG thickness of $2.5 \mu \mathrm{m}$. Moreover, in the effective mode area profile, the fabricated MZM-LN exhibited a higher profile in comparison with the simulated MZM-LN. The mode location and size are high due to the higher refractive index of the fabricated MZM-LN (3.6101) than the one used in the simulated MZM-LN (3.48) [23, 32].

The figure of merit (Vז.L) obtained from the fabricated MZM-LN using both $L N$ and Si substrates as a function of $n_{\text {eff }}$ and Si WG width is presented in Figs. 7(a and b). A linear relation exists between the $n_{\text {eff }}$

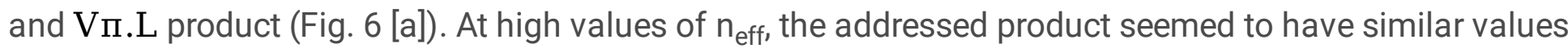
for the three introduced refractive indices (bulk, Si and LN). The product demonstrated a range from 2.12 to 2.25 at a device length of $2 \mathrm{~cm}$, which is within the acceptable Vח.L value that was reported previously [32,33]. The variation of the Vח.L as a function of the Si WG width is shown in Fig. 7(b), in which the obtained values increased along with the increment of the Si WG width. The obtained value of the Vח.L was similar in the bulk and Si profiles, while a slightly higher value was noted in the LN profile; this finding indicates a width of $2.5 \mu \mathrm{m}$, which was the applied width throughout the deposition process of the MZM-LN device. The utilisation of Si substrate indicated a better Vח.L value than that of the LN substrate.

Figure 8 presents the bandwidth of the fabricated MZM-LN at different $\Delta \mathrm{n}$. Our calculation indicates that $\Delta \mathrm{n} \geq 0.132$ provides an acceptable value to achieve $100 \mathrm{Ghz}$ electrical modulation bandwidth. Specifically, the fabricated MZM-LN with $L=2 \mathrm{~cm}$ delivers a bandwidth value at around $50 \mathrm{Ghz}$ with respect to the utilised $\Delta \mathrm{n}[23]$.

\section{Conclusions}


In summary, optimum MZM performance can be obtained using Si WG with a width of $2.5 \mu \mathrm{m}$ and thicknesses of 0.43 and $0.44 \mu \mathrm{m}$ alongside a $\mathrm{SiO}_{2}$ layer of $0.5 \mu \mathrm{m}$ thickness. The development of the $\mathrm{Si}$ substrate obtained a better $\vee \pi . L$ value as compared with the LN substrate with respect to the Si WG width $(2.5 \mu \mathrm{m})$.

\section{Declarations}

\subsection{Funding}

The research reported in this manuscript has been done at the University of Technology, Baghdad, Iraq. Fully funded of our own financial support.

\subsection{Disclosures}

I am the undersigned on behalf of the authors declare that this manuscript is original, has not been published before, and is not currently being considered for publication elsewhere.

Our manuscript creates a paradigm for future studies to encourage researchers to focus on this area. All Authors were aware of about the paper content and approved its submission.

We wish to confirm that there are no known conflicts of interest associated with this publication and there has been no significant financial support for this work that could have influenced its outcome.

We confirm that the manuscript has been read and approved by all named authors and that there are no other persons who satisfied the criteria for authorship but are not listed. We further confirm that the order of authors listed in the manuscript has been approved by all of us.

We confirm that we have given due consideration to the protection of intellectual property associated with this work and that there are no impediments to publication, including the timing of publication, with respect to intellectual property. In so doing we confirm that we have followed the regulations of our institutions concerning intellectual property.

We understand that the Corresponding Author is the sole contact for the Editorial process (including Editorial Manager and direct communications with the office). He is responsible for communicating with the other authors about progress, submissions of revisions, and final approval of proofs.

We confirm that if accepted, the article will not be published elsewhere in the same form, in any language, without the written consent of the publisher.

\section{References}

1. M. A Fakhri, M. J. AbdulRazzaq, A. A. Alwahib, W. H Muttlak," Theoretical study of a pure LinbOn/Ouartz waveauide coated aold nanorods using supercontinuum laser source," Optical Loading [MathJax]/jax/output/CommonHTML/fonts/TeX/fontdata.js 
Materials 109, 110363 (2020).

2. E.L. Wooten, K.M. Kissa, A. Yi-Yan, E.J. Murphy, D.A. Lafaw, P.F. Hallemeier, D. Maack, D.V. Attanasio, D.J. Fritz, G.J. McBrien," A review of lithium niobate modulators for fiber-optic communications systems," IEEE Journal of selected topics in Quantum Electronics 6, 69-82 (2000).

3. D. Janner, D. Tulli, M. García-Granda, M. Belmonte, V. Pruneri," Micro-structured integrated electrooptic $\mathrm{LiNbO}_{3}$ modulators," Laser \& Photonics Reviews 3, 301-313 (2009).

4. C. Zhang, P.A. Morton, J.B. Khurgin, J.D. Peters, J.E. Bowers," Ultralinear heterogeneously integrated ring-assisted Mach-Zehnder interferometer modulator on silicon," Optica 3, 1483-1488 (2016).

5. C. Haffner, W. Heni, Y. Fedoryshyn, J. Niegemann, A. Melikyan, D.L. Elder, B. Baeuerle, Y. Salamin, A. Josten, U. Koch," All-plasmonic Mach-Zehnder modulator enabling optical high-speed communication at the microscale," Nature Photonics 9, 525-528 (2015).

6. J.-H. Han, F. Boeuf, J. Fujikata, S. Takahashi, S. Takagi, M. Takenaka," Efficient low-loss InGaAsP/Si hybrid MOS optical modulator," Nature Photonics 11, 486-490 (2017).

7. N. Kikuchi, E. Yamada, Y. Shibata, H. Ishii," High-speed InP-based Mach-Zehnder modulator for advanced modulation formats," in: 2012 IEEE Compound Semiconductor Integrated Circuit Symposium (CSICS), IEEE, 1-4 (2012).

8. C.T. Phare, Y.-H.D. Lee, J. Cardenas, M. Lipson," Graphene electro-optic modulator with $30 \mathrm{GHz}$ bandwidth," Nature Photonics 9, 511-514 (2015).

9. V. Sorianello, M. Midrio, G. Contestabile, I. Asselberghs, J. Van Campenhout, C. Huyghebaert, I. Goykhman, A. Ott, A. Ferrari, M. Romagnoli," Graphene-silicon phase modulators with gigahertz bandwidth," Nature Photonics 12, 40-44 (2018).

10. M. He, M. Xu, Y. Ren, J. Jian, Z. Ruan, Y. Xu, S. Gao, S. Sun, X. Wen, L. Zhou," High-performance hybrid silicon and lithium niobate Mach-Zehnder modulators for 100 Gbit s- 1 and beyond," Nature Photonics 13, 359-364 (2019).

11. G. Raybon, J. Cho, A. Adamiecki, P. Winzer, A. Konczykowska, F. Jorge, J.-Y. Dupuy, M. Riet, B. Duval, K. Kim," Single carrier high symbol rate transmitter for data rates up to $1.0 \mathrm{~Tb} / \mathrm{s}$," in Optical Fiber Communication Conference, , OSA Technical Digest (online) (Optical Society of America, 2016), paper Th3A.2.

12. S.K. Korotky," Lithium niobate integrated optics: Selected contemporary devices and system applications," Optical Fiber Telecommunications III CH 9, 377 (1997).

13. E.L. Wooten, K.M. Kissa, A. Yi-Yan, E.J. Murphy, D.A. Lafaw, P.F. Hallemeier, D. Maack, D.V. Attanasio, D.J. Fritz, G.J. McBrien,"A review of lithium niobate modulators for fiber-optic communications systems," IEEE Journal of selected topics in Quantum Electronics 6, 69-82 (2000).

14. S. Saha, S.S. Yohanes, D. Jun, A. Danner, M. Tsang,"Fabrication and characterization of optical devices on lithium niobate on insulator chips," Procedia Engineering 140, 183-186 (2016).

15. M. Xu, W. Chen, M. He, X. Wen, Z. Ruan, J. Xu, L. Chen, L. Liu, S. Yu, X. Cai,"Michelson interferometer modulator based on hybrid silicon and lithium niobate platform," APL Photonics 4, 100802 (2019). 
16. C. Wang, M. Zhang, X. Chen, M. Bertrand, A. Shams-Ansari, S. Chandrasekhar, P. Winzer, M. Lončar,"Integrated lithium niobate electro-optic modulators operating at CMOS-compatible voltages," Nature 562, 101-104 (2018).

17. L.Z. Mohammed, M.A. Fakhri, A. Abass,"An overview of optical modulator based on nanophotonic lithium niobate film," AIP Conference Proceedings 2213, 020231 (2020).

18. L. Chen, Q. Xu, M.G. Wood, R.M. Reano,"Hybrid silicon and lithium niobate electro-optical ring modulator," Optica 1,112-118 (2014).

19. S. Jin, L. Xu, H. Zhang, Y. Li,"LiNbO 3 thin-film modulators using silicon nitride surface ridge waveguides," IEEE Photonics Technology Letters 28, 736-739 (2015).

20. P.O. Weigel, J. Zhao, K. Fang, H. Al-Rubaye, D. Trotter, D. Hood, J. Mudrick, C. Dallo, A.T. Pomerene, A.L. Starbuck,"Bonded thin film lithium niobate modulator on a silicon photonics platform exceeding $100 \mathrm{GHz}$ 3-dB electrical modulation bandwidth," Optics express 26, 23728-23739 (2018).

21. P.O. Weigel, High-speed hybrid silicon-lithium niobate electro-optic modulators \& related technologies, ProQuest Dissertations And Theses; Thesis (Ph.D.)--University of California, San Diego, Publication Number: AAT 10814977; ISBN: 9780438168152; Source: Dissertation Abstracts International, 7912(E), Section: B.; 179 (2018).

22. L.Z. Mohammed, M.A. Fakhri, A. Abass,"Structural and Optical Properties of nanostructured hybrid LiNb03/Silicon wafer for Fabricating Optical Modulator," J. Phys.: Conf. Ser. 1795, 012055 (2021).

23. M. A. Fakhri, Y. Al-Douri, U. Hashim, E. T. Salim, D. Prakash, K. D. Verma," Optical investigation of nanophotonic lithium niobate-based optical waveguide," Applied Physics B: Lasers and Optics 121(1) , 107-116 (2015).

24. M. A.Fakhri, U.Hashim, E. T.Salim, Z. T. Salim," Preparation and charactrization of photonic $\mathrm{LiNbO}_{3}$ generated from mixing of new raw materials using spry pyrolysis method," Journal of Materials Science: Materials in Electronics 27(12), 13105-13112 (2016).

25. L.Z. Mohammed, M.A. Fakhri, A. Abass,"The Effect of Mode Area and Refractive Index for Optical TE Mode Propagation in Hybrid LN/Si Electro-Optic Structure of Mach-Zehnder Modulator," Iraqi Jounal of Computers, Communication, Control \& Systems Engineering 20, 71-77 (2020).

26. E.T. Salim, J.A. Saimon, M.K. Abood, M.A. Fakhri," Effect of silicon substrate type on $\mathrm{Nb}_{2} \mathrm{O}_{5} /$ Si device performance: an answer depends on physical analysis," Optical and Quantum Electronics 52(10), 463 (2020).

27. ET Salim, MA Fakhri, Z Tareq, U Hashim," Electrical and electronic properties of lithium based thin film for photonic application," AIP Conference Proceedings 2213 (1), 020230 (2020).

28. R.A. Soomro, S.H. Sherazi, N. Memon, M. Shah, N. Kalwar, K.R. Hallam, A. Shah," Synthesis of air stable copper nanoparticles and their use in catalysis," Adv. Mater. Lett, 5, 191-198 (2014).

29. MA Fakhri, ET Salim, MHA Wahid, ZT Salim, U Hashim," A novel parameter effects on optical properties of the $\mathrm{LiNbO}_{3}$ films using sol-gel method,"AIP Conference Proceedings 2213(1), 020242 (2020).

Loading [MathJax]/jax/output/CommonHTML/fonts/TeX/fontdata.js 
30. MT Awayiz, ET Salim, Silver oxide nanoparticle," effect of chemical interaction temperatures on structural properties and surface roughness," AIP Conference Proceedings 2213 (1), 020247 (2020).

31. H. Han, B. Xiang, T. Lin, G. Chai, S. Ruan,"Design and Optimization of Proton Exchanged Integrated Electro-Optic Modulators in X-Cut Lithium Niobate Thin Film," Crystals 9, 549 (2019).

32. S. Sun, M. He, S. Yu, and X. Cai, "Hybrid Silicon and Lithium Niobate Mach-Zehnder Modulators with High Bandwidth Operating at C-band and O-band," in Conference on Lasers and Electro-Optics, OSA Technical Digest (Optical Society of America, 2020), paper STh1F.4.

33. H. Han, B. Xiang,"Integrated electro-optic modulators in x-cut lithium niobate thin film, Optik 212, 164691 (2020).

\section{Figures}

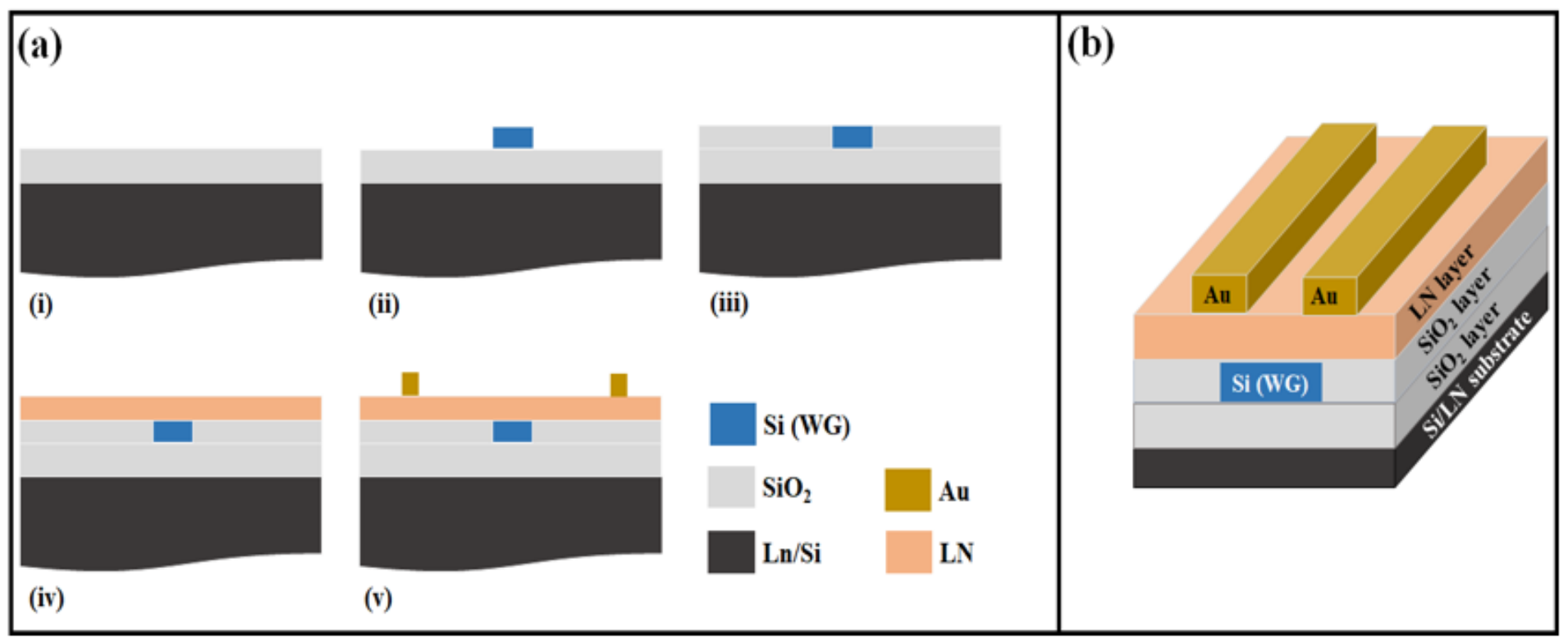

Figure 1

Schematic illustration of the Device fabrication process deposited layer by layer of MZM, and (b) 3D schematic of a hybrid Si/LN substrate 

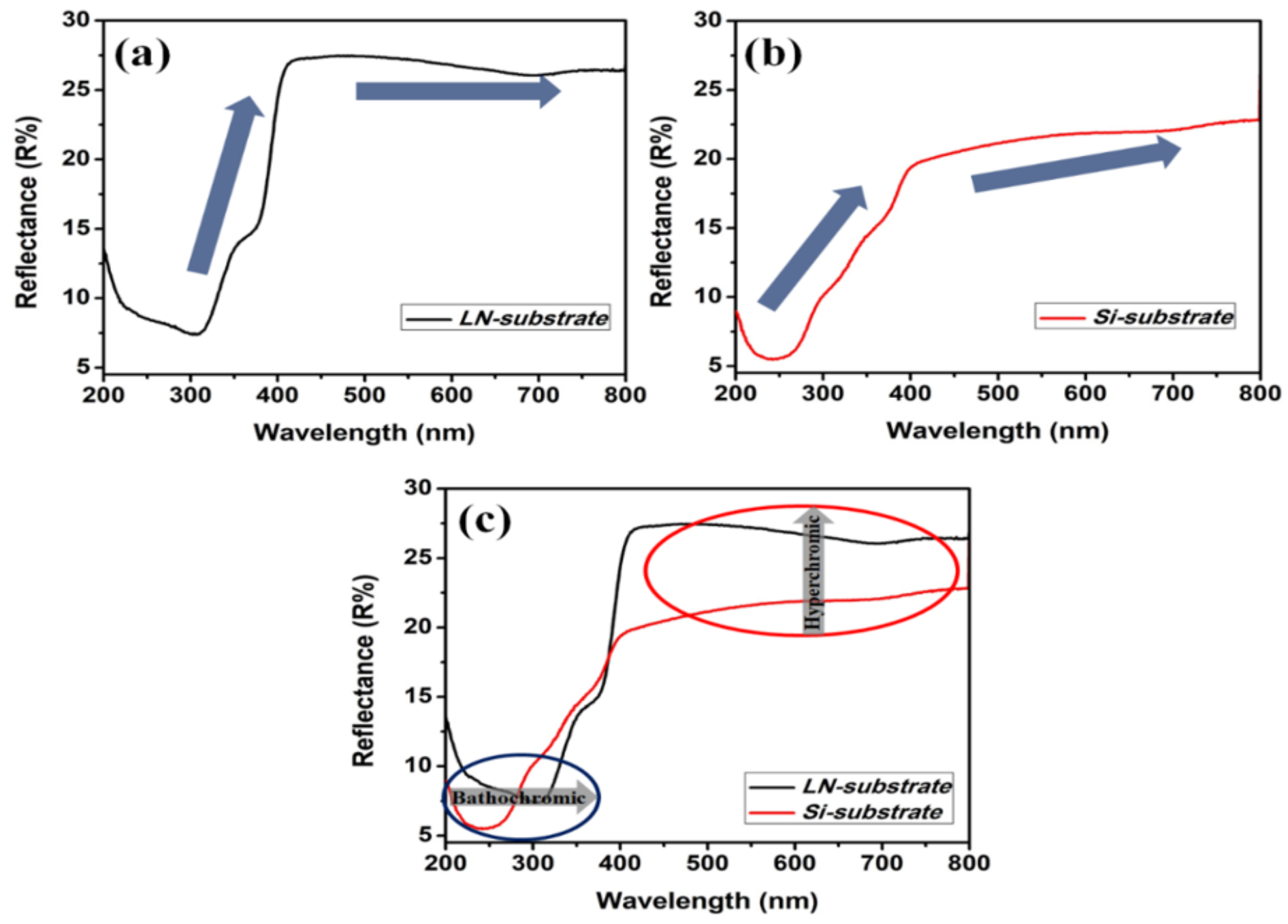

Figure 2

Reflectance spectra of the LN deposited films; (a) LN-substrate, (b) Si-substrate, and (c) comparison n between the two deposited films.
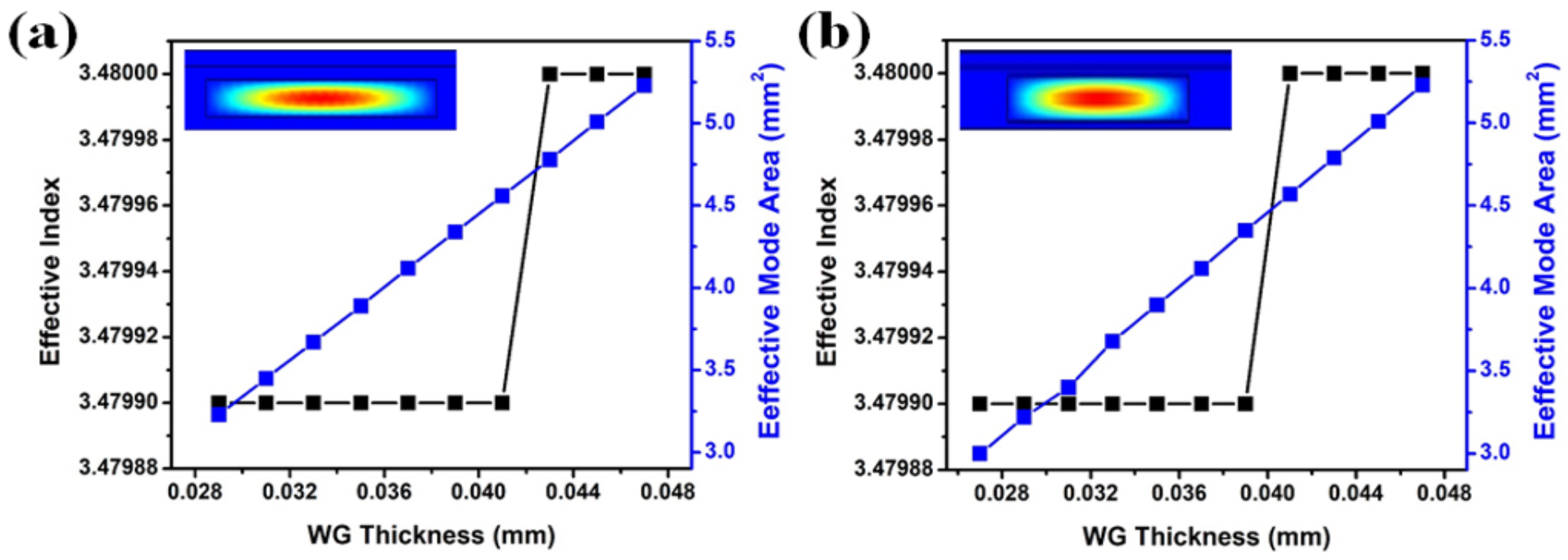
Effective index and effective mode area of the simulated MZM at SiO2 thickness of 0.5mm; (a) MZM and (b) MZM-LN.
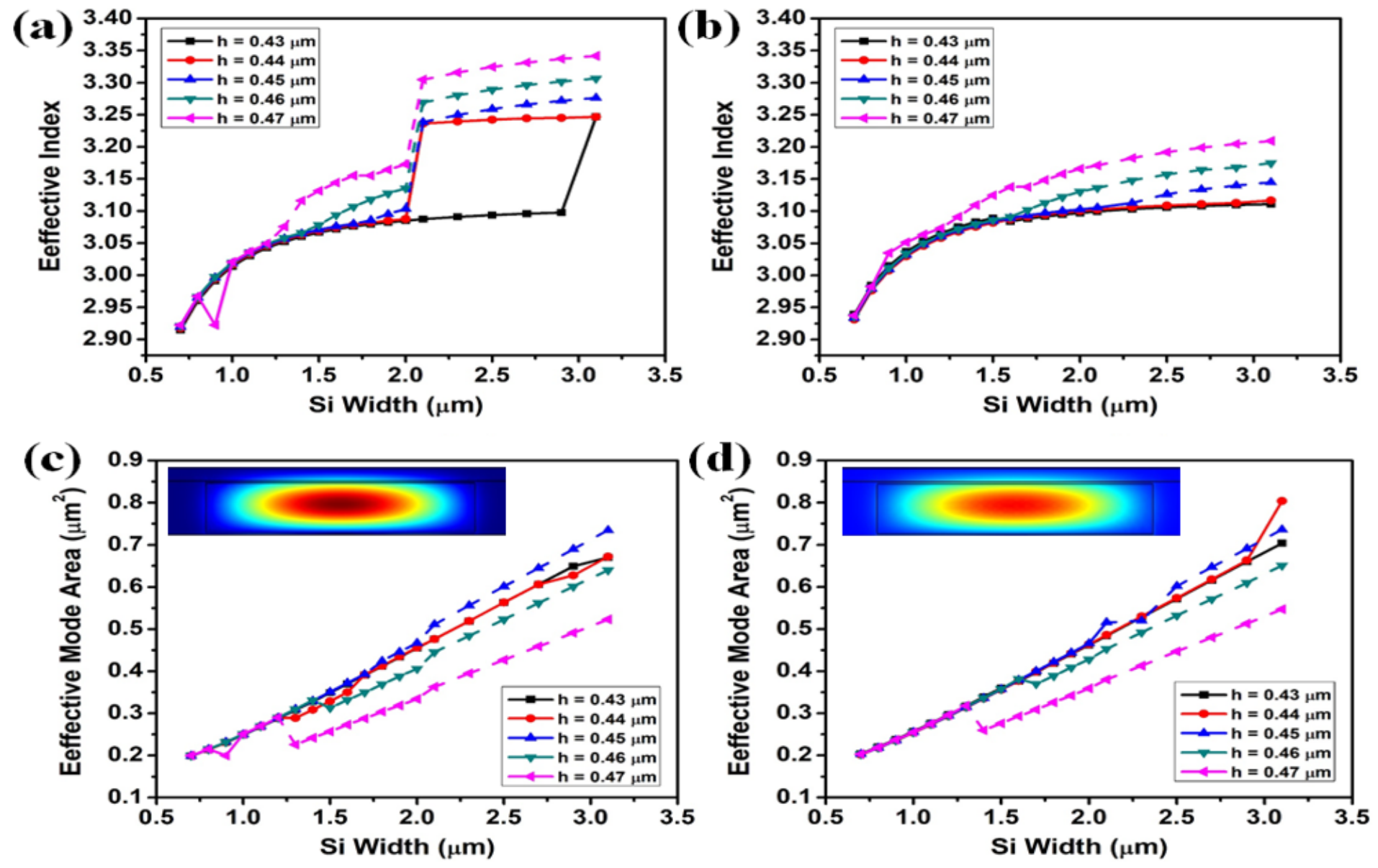

Figure 4

Variation of effective index and effective mode area as a function of Si WG width for different thicknesses with SiO2 layer thickness of $0.5 \mu \mathrm{m}$; (a) MZM, (b) MZM-LN, (c) MZM, and (d) MZM-LN. 

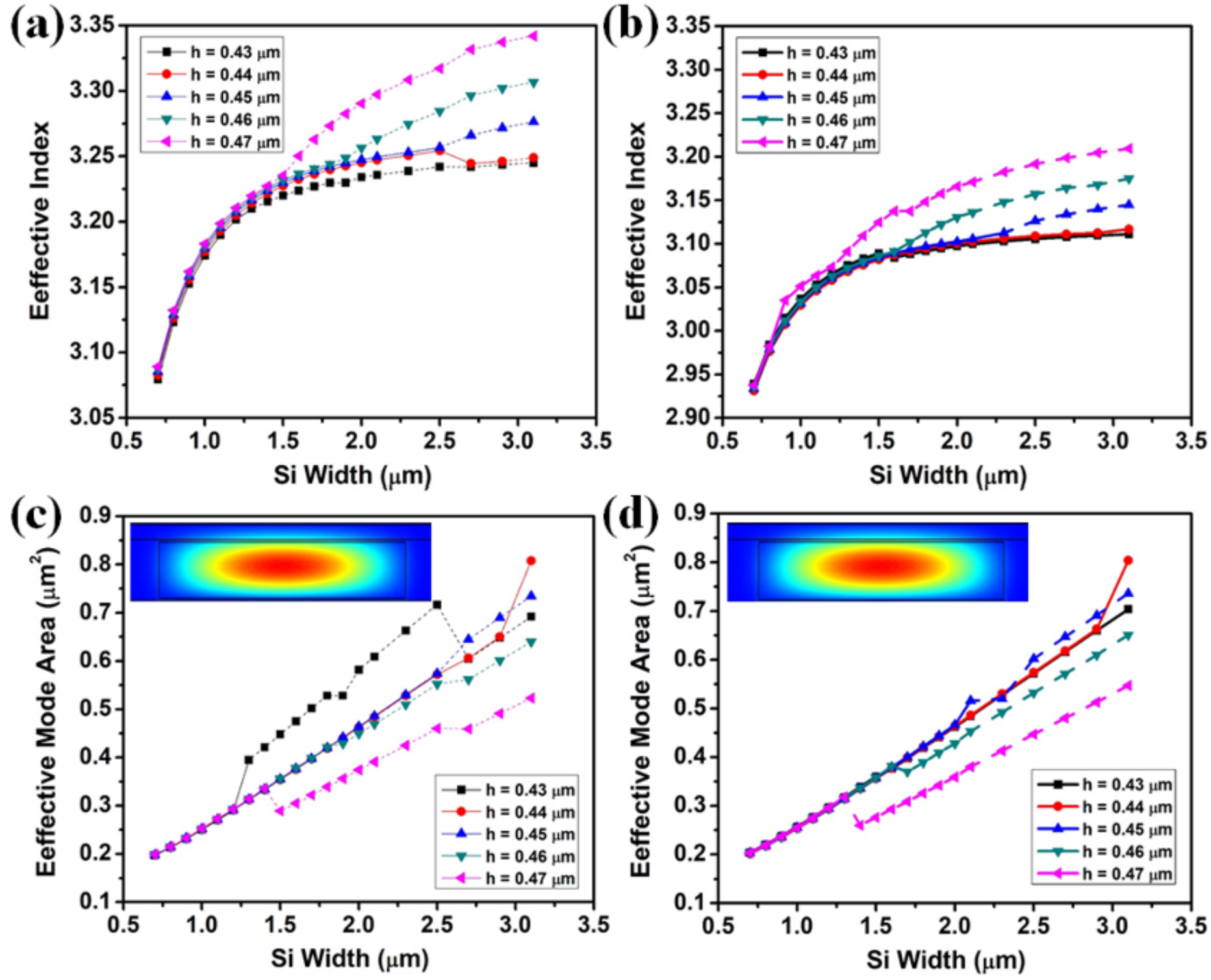

Figure 5

Variation of effective index (a) Fabricated MZM-LN and (b) simulated MZM-LN and effective mode area (c) fabricated MZM-LN and (d) simulated MZM-LN, using LN substrate. 

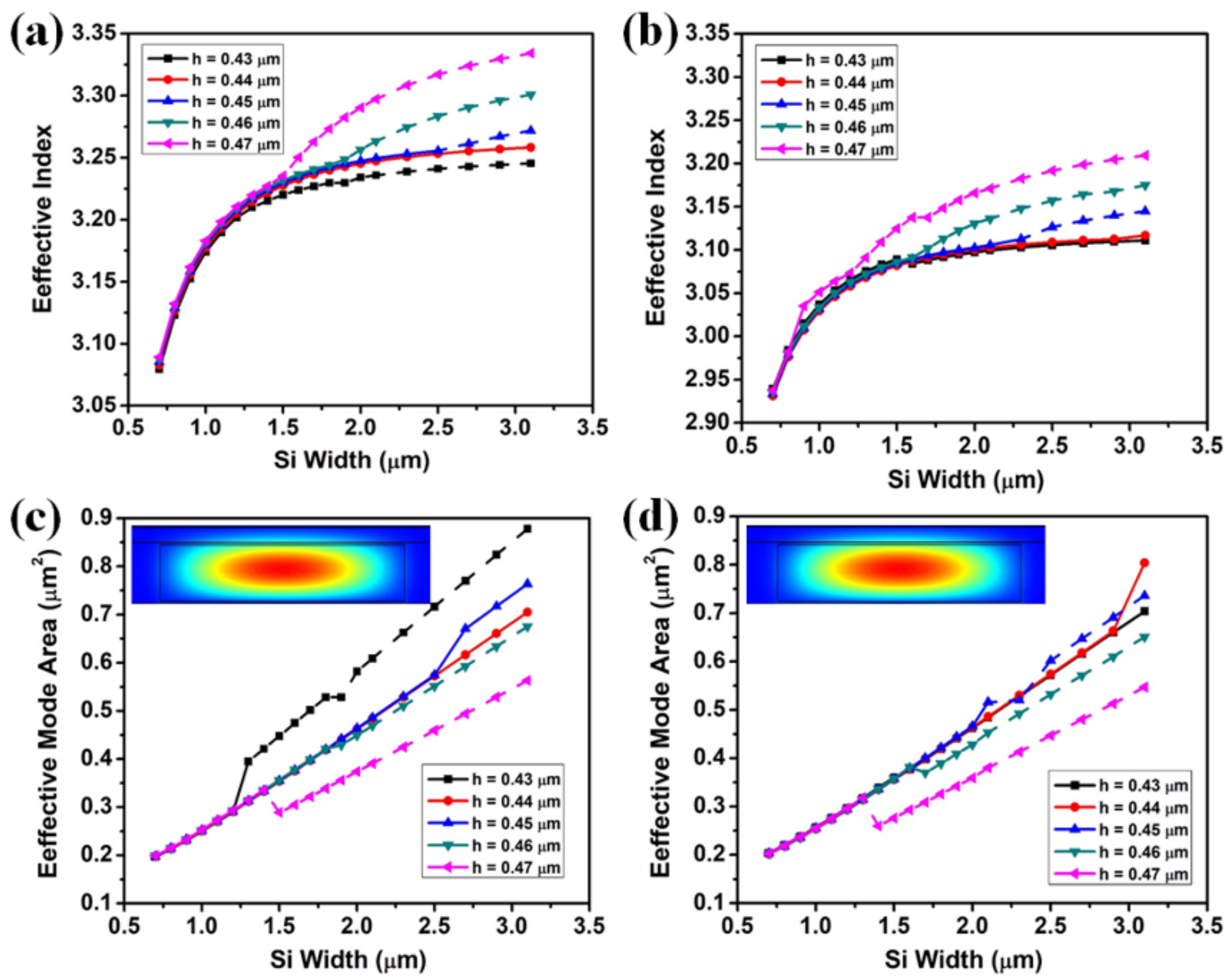

Figure 6

Variation of effective index (a) Fabricated MZM-LN and (b) simulated MZM-LN and effective mode area (c) fabricated MZM-LN and (d) simulated MZM-LN, using Si substrate. 

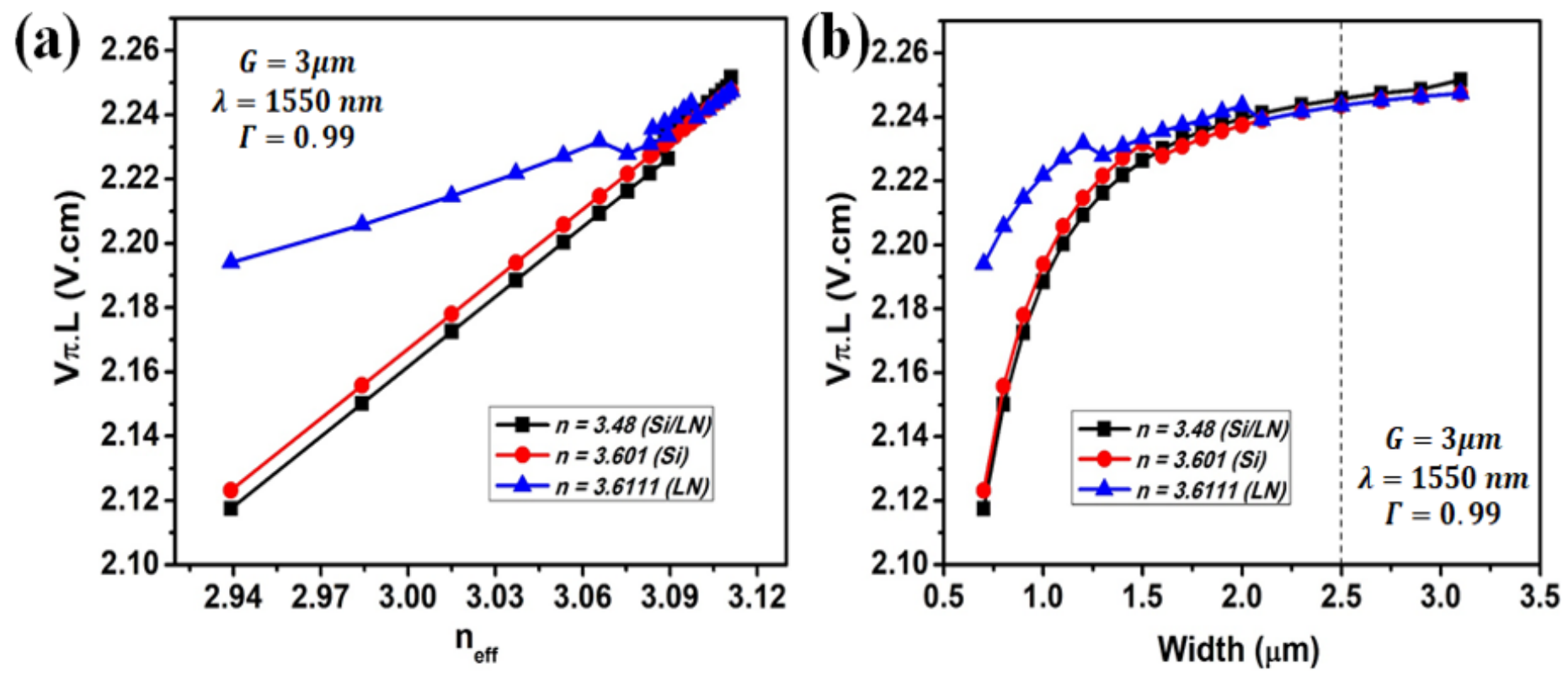

Figure 7

Half-wave voltage-length product of the fabricated MZM-LN variation with (a) neff, (b) Si WG width.

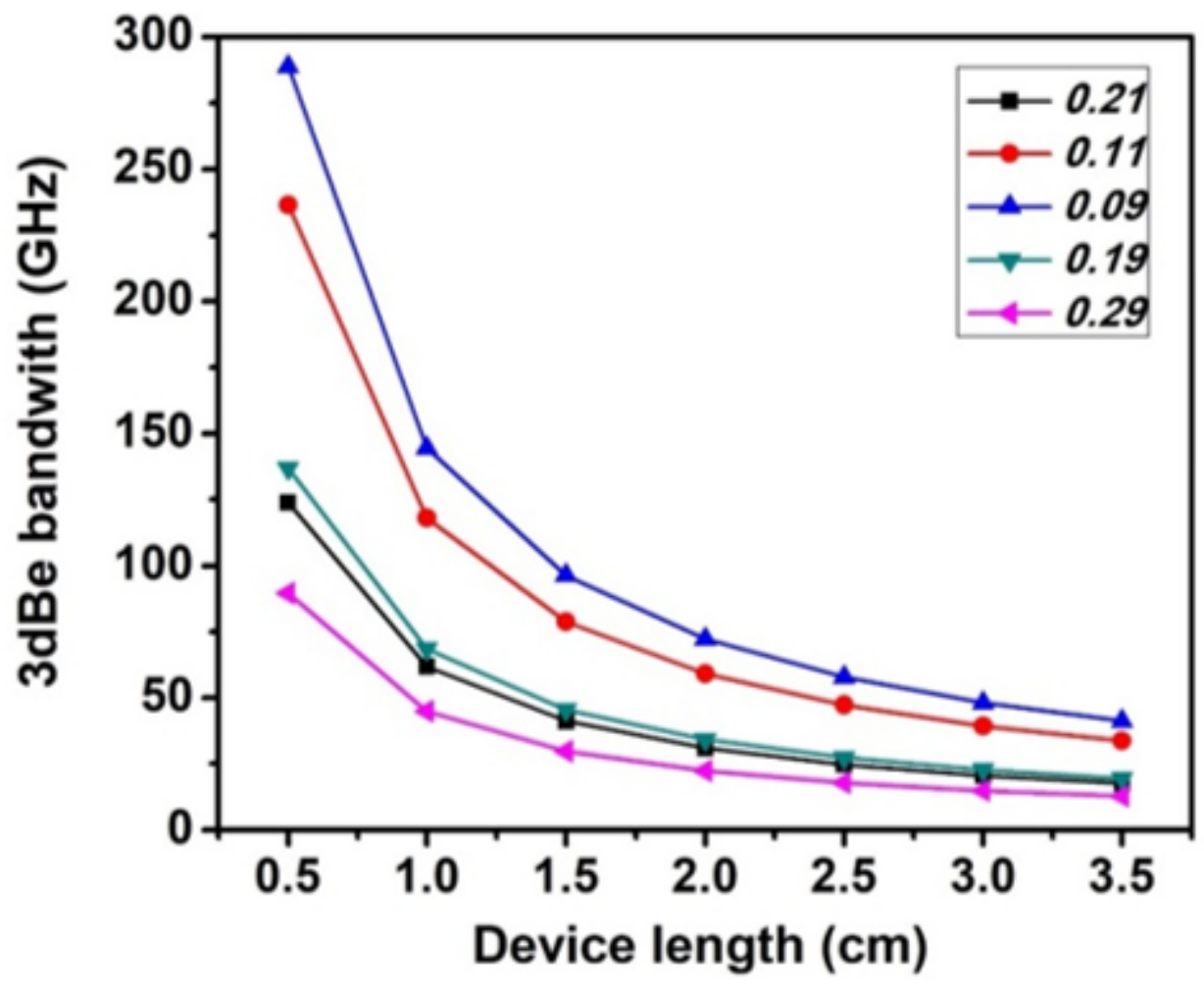

Figure 8

The fabricated MZM-LN 3-dBe electrical bandwidth values against the device length. 